\title{
Septic analogues of the Rogers-Ramanujan functions
}

\author{
by \\ HeEkyoung Hahn (Urbana, IL)
}

1. Introduction. The well known Rogers-Ramanujan functions are defined for $|q|<1$ by

$$
G(q):=\sum_{n=0}^{\infty} \frac{q^{n^{2}}}{(q ; q)_{n}}, \quad H(q):=\sum_{n=0}^{\infty} \frac{q^{n^{2}+n}}{(q ; q)_{n}} .
$$

These functions satisfy the famous Rogers-Ramanujan identities [8, pp. 214215]

$$
G(q)=\frac{1}{\left(q ; q^{5}\right)_{\infty}\left(q^{4} ; q^{5}\right)_{\infty}}, \quad H(q)=\frac{1}{\left(q^{2} ; q^{5}\right)_{\infty}\left(q^{3} ; q^{5}\right)_{\infty}},
$$

where, as usual, for any complex number $a,(a ; q)_{0}=1$, for $n \geq 1$,

$$
(a ; q)_{n}:=\prod_{k=1}^{n}\left(1-a q^{k-1}\right), \quad(a ; q)_{\infty}=\prod_{k=1}^{\infty}\left(1-a q^{k-1}\right) .
$$

At the end of his brief communication [7], [8, p. 231] announcing his proofs of the Rogers-Ramanujan identities (1.2), Ramanujan remarks, "I have now found an algebraic relation between $G(q)$ and $H(q)$, viz.:

$$
H(q)\{G(q)\}^{11}-q^{2} G(q)\{H(q)\}^{11}=1+11 q\{G(q) H(q)\}^{6} .
$$

Another noteworthy formula is

$$
H(q) G\left(q^{11}\right)-q^{2} G(q) H\left(q^{11}\right)=1 .
$$

Each of these formulae is the simplest of a large class." In a manuscript of Ramanujan, published with his Lost Notebook [9], there are forty identities involving the Rogers-Ramanujan functions.

In 1921, L. J. Rogers [10] established ten of the identities including (1.3) and (1.4). In his paper [12] proving eight of the identities, but with two of them from the list that Rogers proved, Watson confides, "Among the formulae contained in the manuscripts left by Ramanujan is the set of about forty which involve functions of the types $G(q)$ and $H(q)$; the beauty of

2000 Mathematics Subject Classification: Primary 33D90, 11P83. 
these formulae seems to me comparable with that of the Rogers-Ramanujan identities."

Ramanujan's forty identities for $G(q)$ and $H(q)$ were first brought to the mathematical public by B. J. Birch [3] in 1975. As mentioned above, Rogers [10] first established ten of them. After Watson's paper, D. Bressoud [5] proved fifteen from the list of forty in 1977. The remaining nine identities were proved by A. Biagioli [2] using modular forms.

Now, let us define the septic Rogers-Ramanujan type functions,

$$
\begin{aligned}
& A(q):=\sum_{n=0}^{\infty} \frac{q^{2 n^{2}}}{\left(q^{2} ; q^{2}\right)_{n}(-q ; q)_{2 n}}=\frac{\left(q^{7} ; q^{7}\right)_{\infty}\left(q^{3} ; q^{7}\right)_{\infty}\left(q^{4} ; q^{7}\right)_{\infty}}{\left(q^{2} ; q^{2}\right)_{\infty}} \\
& B(q):=\sum_{n=0}^{\infty} \frac{q^{2 n(n+1)}}{\left(q^{2} ; q^{2}\right)_{n}(-q ; q)_{2 n}}=\frac{\left(q^{7} ; q^{7}\right)_{\infty}\left(q^{2} ; q^{7}\right)_{\infty}\left(q^{5} ; q^{7}\right)_{\infty}}{\left(q^{2} ; q^{2}\right)_{\infty}} \\
& C(q):=\sum_{n=0}^{\infty} \frac{q^{2 n(n+1)}}{\left(q^{2} ; q^{2}\right)_{n}(-q ; q)_{2 n+1}}=\frac{\left(q^{7} ; q^{7}\right)_{\infty}\left(q ; q^{7}\right)_{\infty}\left(q^{6} ; q^{7}\right)_{\infty}}{\left(q^{2} ; q^{2}\right)_{\infty}}
\end{aligned}
$$

where the latter equalities are due to L. J. Slater [11].

The main purpose of this paper is to establish several analogues of Ramanujan's forty identities involving $A(q), B(q)$, and $C(q)$. Some of these identities that we found have partition-theoretic interpretations. In the final section, some results related to the theory of partitions are established by introducing the notion of colored partitions.

2. Definitions and preliminary results. We first recall Ramanujan's definition for a general theta function

$$
f(a, b)=\sum_{n=-\infty}^{\infty} a^{n(n+1) / 2} b^{n(n-1) / 2}, \quad|a b|<1 .
$$

Then it is easy to verify that $[1$, p. 34 , Entry 18]

$$
\begin{aligned}
f(a, b) & =f(b, a), \\
f(1, a) & =2 f\left(a, a^{3}\right), \\
f(-1, a) & =0,
\end{aligned}
$$

and by Jacobi triple product identity [1, p. 35, Entry 19], we have

$$
f(a, b)=(-a ; a b)_{\infty}(-b ; a b)_{\infty}(a b, a b)_{\infty} .
$$

Another basic property satisfied by $f(a, b)$ is that, for any integer $n$,

$$
f(a, b)=a^{n(n+1) / 2} b^{n(n-1) / 2} f\left(a(a b)^{n}, b(a b)^{-n}\right) .
$$


The three most important special cases of (2.1) are

$$
\begin{aligned}
\varphi(q) & :=f(q, q)=\sum_{n=-\infty}^{\infty} q^{n^{2}}=\left(-q ; q^{2}\right)_{\infty}^{2}\left(q^{2} ; q^{2}\right)_{\infty}, \\
\psi(q) & :=f\left(q, q^{3}\right)=\sum_{n=0}^{\infty} q^{n(n+1) / 2}=\frac{\left(q^{2} ; q^{2}\right)_{\infty}}{\left(q ; q^{2}\right)_{\infty}} \\
f(-q) & :=f\left(-q ;-q^{2}\right)=\sum_{n=-\infty}^{\infty}(-1)^{n} q^{n(3 n-1) / 2} \\
& =(q ; q)_{\infty}=: q^{-1 / 24} \eta(\tau),
\end{aligned}
$$

where $q=e^{2 \pi i \tau}, \operatorname{Im} \tau>0$, and $\eta$ denotes the Dedekind eta function. Also, after Ramanujan, define

$$
\chi(q):=\left(-q ; q^{2}\right)_{\infty}
$$

It is easy to show that

$$
\begin{gathered}
\chi(q)=\frac{f(q)}{f\left(-q^{2}\right)}=\frac{\varphi(q)}{f(q)}=\frac{f\left(-q^{2}\right)}{\psi(-q)}, \\
\chi(q) \chi(-q)=\chi\left(-q^{2}\right) .
\end{gathered}
$$

The function $f(a, b)$ also satisfies a useful addition formula. For each positive integer $n$, let

$$
U_{n}:=a^{n(n+1) / 2} b^{n(n-1) / 2}, \quad V_{n}:=a^{n(n-1) / 2} b^{n(n+1) / 2} .
$$

Then [1, p. 48, Entry 31]

$$
f\left(U_{1}, V_{1}\right)=\sum_{r=0}^{n-1} U_{r} f\left(\frac{U_{n+r}}{U_{r}}, \frac{V_{n-r}}{U_{r}}\right) .
$$

Using (2.5), we can rewrite our septic identities (1.5)-(1.7) in the forms

$$
A(q)=\frac{f\left(-q^{3},-q^{4}\right)}{f\left(-q^{2}\right)}, \quad B(q)=\frac{f\left(-q^{2},-q^{5}\right)}{f\left(-q^{2}\right)}, \quad C(q)=\frac{f\left(-q,-q^{6}\right)}{f\left(-q^{2}\right)} .
$$

We shall use (2.14) many times in the remainder of the paper.

3. Main results. In this section, we present a list of modular relations for $A(q), B(q)$, and $C(q)$, which we prove in this paper. These identities (3.1)-(3.29) involve $A(q), B(q)$, and $C(q)$ in the combinations of

$$
\begin{gathered}
A\left(q^{\beta}\right) A\left(q^{\alpha}\right)+q^{(\alpha+\beta) / 7} B\left(q^{\beta}\right) B\left(q^{\alpha}\right)+q^{(3 \alpha+3 \beta) / 7} C\left(q^{\beta}\right) C\left(q^{\alpha}\right), \\
A\left(q^{\beta}\right) B\left(q^{\alpha}\right)-q^{(2 \alpha+\beta) / 7} B\left(q^{\beta}\right) C\left(q^{\alpha}\right)-q^{(-\alpha+3 \beta) / 7} C\left(q^{\beta}\right) A\left(q^{\alpha}\right), \\
A\left(q^{\beta}\right) C\left(q^{\alpha}\right)-q^{(-3 \alpha+\beta) / 7} B\left(q^{\beta}\right) A\left(q^{\alpha}\right)+q^{(-2 \alpha+3 \beta) / 7} C\left(q^{\beta}\right) B\left(q^{\alpha}\right),
\end{gathered}
$$

where $\alpha$ and $\beta$ are positive integers. 
From now on, for positive integer $n$, let

$$
A_{n}:=A\left(q^{n}\right), \quad B_{n}:=B\left(q^{n}\right), \quad C_{n}:=C\left(q^{n}\right),
$$

and

$$
A_{n}^{*}:=A\left(-q^{n}\right), \quad B_{n}^{*}:=B\left(-q^{n}\right), \quad C_{n}^{*}:=C\left(-q^{n}\right) .
$$

We will prove that

$$
\begin{aligned}
& A_{1} B_{3}-q B_{1} C_{3}-C_{1} A_{3}=0, \\
& A_{6} A_{1}+q B_{6} B_{1}+q^{3} C_{6} C_{1}=\frac{\chi^{3}\left(-q^{3}\right) \chi\left(q^{3}\right)}{\chi(-q)}, \\
& A_{3} B_{2}-q B_{3} C_{2}-q C_{3} A_{2}=\frac{\chi^{3}(-q) \chi(q)}{\chi\left(-q^{3}\right)}, \\
& A_{10} C_{1}-q B_{10} A_{1}+q^{4} C_{10} B_{1}=\chi^{2}(-q) \chi\left(q^{5}\right), \\
& A_{5} A_{2}+q B_{5} B_{2}+q^{3} C_{5} C_{2}=\chi^{2}\left(-q^{5}\right) \chi(q) \text {, } \\
& A_{12} B_{1}-q^{2} B_{12} C_{1}-q^{5} C_{12} A_{1}=\chi\left(-q^{2}\right) \chi\left(q^{3}\right) \chi\left(-q^{12}\right) \text {, } \\
& A_{4} A_{3}+q B_{4} B_{3}+q^{3} C_{4} C_{3}=\chi(q) \chi\left(-q^{4}\right) \chi\left(-q^{6}\right), \\
& A_{6}^{*} A_{1}^{*}+q B_{6}^{*} B_{1}^{*}+q^{3} C_{6}^{*} C_{1}^{*}=\frac{\chi\left(-q^{12}\right)}{\chi(-q)}, \\
& A_{3}^{*} B_{2}^{*}+q B_{3}^{*} C_{2}^{*}-q C_{3}^{*} A_{2}^{*}=\frac{\chi\left(-q^{4}\right)}{\chi\left(-q^{3}\right)}, \\
& A_{10}^{*} C_{1}^{*}-q B_{10}^{*} A_{1}^{*}-q^{4} C_{10}^{*} B_{1}^{*}=\chi\left(q^{2}\right) \chi\left(q^{5}\right), \\
& A_{5}^{*} A_{2}^{*}+q B_{5}^{*} B_{2}^{*}+q^{3} C_{5}^{*} C_{2}^{*}=\chi(q) \chi\left(q^{10}\right), \\
& A_{12}^{*} B_{1}^{*}+q^{2} B_{12}^{*} C_{1}^{*}-q^{5} C_{12}^{*} A_{1}=\frac{\chi\left(q^{3}\right) \chi\left(q^{4}\right) \chi\left(-q^{12}\right)}{\chi\left(-q^{2}\right)}, \\
& A_{4}^{*} A_{3}^{*}+q B_{4}^{*} B_{3}^{*}+q^{3} C_{4}^{*} C_{3}^{*}=\frac{\chi(q) \chi\left(-q^{4}\right) \chi\left(q^{12}\right)}{\chi\left(-q^{6}\right)}, \\
& A_{5} B_{1}-q B_{5} C_{1}-q^{2} C_{5} A_{1}=\chi(-q) \chi\left(-q^{5}\right), \\
& A_{17} C_{1}-q^{2} B_{17} A_{1}+q^{7} C_{17} B_{1}=\chi(-q) \chi\left(-q^{17}\right), \\
& A_{19} B_{1}-q^{3} B_{19} C_{1}-q^{8} C_{19} A_{1} \\
& =-\frac{q^{2}}{\chi(-q) \chi\left(-q^{19}\right)}+\frac{1}{4 \sqrt{q}} \chi(-q) \chi\left(-q^{19}\right) \chi^{2}\left(q^{1 / 2}\right) \chi^{2}\left(q^{19 / 2}\right) \\
& -\frac{1}{4 \sqrt{q}} \chi(-q) \chi\left(-q^{19}\right) \chi^{2}\left(-q^{1 / 2}\right) \chi^{2}\left(-q^{19 / 2}\right) \text {, } \\
& A_{20} A_{1}+q^{3} B_{20} B_{1}+q^{9} C_{20} C_{1}=\frac{\chi\left(-q^{5}\right) \chi\left(-q^{10}\right) \chi\left(-q^{20}\right)}{\chi\left(-q^{2}\right)}, \\
& A_{4} B_{5}-q^{2} B_{4} C_{5}-q C_{4} A_{5}=\frac{\chi(-q) \chi\left(-q^{2}\right) \chi\left(-q^{4}\right)}{\chi\left(-q^{10}\right)},
\end{aligned}
$$




$$
\begin{gathered}
A_{24} C_{1}-q^{3} B_{24} A_{1}+q^{10} C_{24} B_{1}=\frac{\chi\left(-q^{6}\right) \chi\left(-q^{24}\right)}{\chi(q)}, \\
A_{8} B_{3}-q^{2} B_{8} C_{3}-q^{3} C_{8} A_{3}=\frac{\chi\left(-q^{2}\right) \chi\left(-q^{8}\right)}{\chi\left(q^{3}\right)}, \\
A_{26} B_{1}-q^{4} B_{26} C_{1}-q^{11} C_{26} A_{1}=\chi\left(-q^{13}\right) \chi\left(-q^{26}\right), \\
A_{13} C_{2}-q B_{13} A_{2}+q^{5} C_{13} B_{2}=\chi(-q) \chi\left(-q^{2}\right), \\
A_{27} A_{1}+q^{4} B_{27} B_{1}+q^{12} C_{27} C_{1}=\frac{f\left(-q^{3}\right) f\left(-q^{9}\right)}{f\left(-q^{2}\right) f\left(-q^{54}\right)}, \\
A_{31} C_{1}-q^{4} B_{31} A_{1}+q^{3} C_{31} B_{1} \\
=\frac{1}{2 q} \chi\left(-q^{2}\right) \chi\left(-q^{62}\right)-\frac{1}{2 q} \chi^{2}(-q) \chi^{2}\left(-q^{31}\right)+\frac{q^{3}}{\chi(q) \chi\left(q^{31}\right)}, \\
A_{40} B_{1}-q^{6} B_{40} C_{1}-q^{17} C_{40} A_{1}=\frac{\chi\left(-q^{5}\right) \chi\left(-q^{20}\right) \chi\left(-q^{40}\right)}{\chi\left(-q^{4}\right)}, \\
A_{16} C_{3}-q B_{16} A_{3}+q^{6} C_{16} B_{3}=\frac{\chi(-q) \chi\left(-q^{4}\right) \chi\left(-q^{8}\right) \chi\left(-q^{16}\right)}{\chi\left(-q^{6}\right) \chi\left(-q^{24}\right)}, \\
A_{48} A_{1}+q^{7} B_{48} B_{1}+q^{21} C_{48} C_{1}=\frac{\chi\left(-q^{3}\right) \chi\left(-q^{12}\right) \chi\left(-q^{24}\right) \chi\left(-q^{48}\right)}{\chi\left(-q^{2}\right) \chi\left(-q^{8}\right)}, \\
A_{171} C_{1}-q^{24} B_{171} A_{1}+q^{73} C_{171} B_{1}=0, \\
A_{195} A_{1}+q^{28} B_{195} B_{1}+q^{84} C_{195} C_{1}=0 .
\end{gathered}
$$

4. Proofs of (3.1)-(3.7). In this section, we will prove relations (3.1)(3.7) by using ideas similar to those of Watson [12]. In all proofs, one expresses the left sides of the identities in terms of theta functions by using (2.14). After clearing fractions, we see that the right side can be expressed as a product of two theta functions, say with summation indices $m$ and $n$. One then tries to find a change of indices of the form

$$
\alpha m+\beta n=7 M+a, \quad \gamma m+\delta n=7 N+b,
$$

so that the product on the right side decomposes into the requisite sum of two products of theta functions on the left side.

Theorem 4.1. The identity (3.1) holds.

Proof. Using (2.14), we may write (3.1) in the alternative form

$$
\begin{aligned}
f\left(-q^{3},-q^{4}\right) f\left(-q^{6},-q^{15}\right)-q f( & \left.-q^{2},-q^{5}\right) f\left(-q^{3},-q^{18}\right) \\
& -f\left(-q,-q^{6}\right) f\left(-q^{9},-q^{12}\right)=0 .
\end{aligned}
$$

Set

$$
2 m+3 n=7 M+a, \quad m-2 n=7 N+b,
$$


where $a$ and $b$ will have values from the set $\{0, \pm 1, \pm 2, \pm 3\}$. Then

$$
m=2 M+3 N+(2 a+3 b) / 7, \quad n=M-2 N+(a-2 b) / 7 .
$$

It follows that the values of $a$ and $b$ are associated as in the following table:

$$
\begin{array}{lllll}
\hline a & 0 & \pm 1 & \pm 2 & \pm 3 \\
b & 0 & \mp 3 & \pm 1 & \mp 2 \\
\hline
\end{array}
$$

When $a$ assumes the values $-3,-2,-1,0,1,2,3$ in succession, it is easy to see that the corresponding values of $m^{2}+m+3 n^{2}+3 n$ are respectively

$$
\begin{aligned}
& 7 M^{2}-M+21 N^{2}+9 N, \\
& 7 M^{2}+M+21 N^{2}-9 N, \\
& 7 M^{2}+3 M+21 N^{2}+15 N+2, \\
& 7 M^{2}+5 M+21 N^{2}-3 N, \\
& 7 M^{2}+7 M+21 N^{2}-21 N+6, \\
& 7 M^{2}+9 M+21 N^{2}+3 N+2, \\
& 7 M^{2}+11 M+21 N^{2}-15 N+6 .
\end{aligned}
$$

Hence, by (2.4),

$$
\begin{aligned}
& f\left(-1,-q^{3}\right) f(-1,-q)=\sum_{m, n=-\infty}^{\infty}(-1)^{m+n} q^{m(m+1) / 2+3 n(n+1) / 2} \\
&=-f\left(-q^{3},-q^{4}\right) f\left(-q^{6},-q^{15}\right)-f\left(-q^{3},-q^{4}\right) f\left(-q^{6},-q^{15}\right) \\
&+q f\left(-q^{2},-q^{5}\right) f\left(-q^{3},-q^{18}\right)+f\left(-q,-q^{6}\right) f\left(-q^{9},-q^{12}\right) \\
&+q^{3} f\left(-1,-q^{7}\right) f\left(-1,-q^{21}\right)-q f\left(-q^{-1},-q^{8}\right) f\left(-q^{9},-q^{12}\right) \\
&-q^{3} f\left(-q^{9},-q^{-2}\right) f\left(-q^{3},-q^{18}\right) \\
&=-2 f\left(-q^{3},-q^{4}\right) f\left(-q^{6},-q^{15}\right)+2 q f\left(-q^{2},-q^{5}\right) f\left(-q^{3},-q^{18}\right) \\
&+2 f\left(-q,-q^{6}\right) f\left(-q^{9},-q^{12}\right)
\end{aligned}
$$

which is the desired identity upon dividing by 2 .

Theorem 4.2. The identity (3.2) holds.

Proof. By using (2.14), we find that (3.2) is equivalent to the identity

$$
\begin{aligned}
f\left(-q^{18},\right. & \left.-q^{24}\right) f\left(-q^{3},-q^{4}\right)+q f\left(-q^{12},-q^{30}\right) f\left(-q^{2},-q^{5}\right) \\
& +q^{3} f\left(-q^{6},-q^{36}\right) f\left(-q,-q^{6}\right)=f\left(-q^{2}\right) f\left(-q^{12}\right) \frac{\chi^{3}\left(-q^{3}\right) \chi\left(q^{3}\right)}{\chi(-q)} .
\end{aligned}
$$

Using (2.11) and (2.12), we find that

$$
f\left(-q^{2}\right) f\left(-q^{12}\right) \frac{\chi^{3}\left(-q^{3}\right) \chi\left(q^{3}\right)}{\chi(-q)}=f\left(-q^{2}\right) f\left(-q^{12}\right) \frac{\chi^{2}\left(-q^{3}\right) \chi\left(-q^{6}\right)}{\chi(-q)}
$$




$$
\begin{aligned}
& =\psi(q) f\left(-q^{12}\right) \chi\left(-q^{6}\right) \chi^{2}\left(-q^{3}\right)=\psi(q) f\left(-q^{6}\right) \chi^{2}\left(-q^{3}\right) \\
& =\psi(q) f\left(-q^{3}\right) \chi\left(-q^{3}\right)=\psi(q) \varphi\left(-q^{3}\right) .
\end{aligned}
$$

It thus suffices to show that

$$
\begin{aligned}
f\left(-q^{18},-q^{24}\right) f\left(-q^{3}\right. & \left.,-q^{4}\right)+q f\left(-q^{12},-q^{30}\right) f\left(-q^{2},-q^{5}\right) \\
& +q^{3} f\left(-q^{6},-q^{36}\right) f\left(-q,-q^{6}\right)=\psi(q) \varphi\left(-q^{3}\right) .
\end{aligned}
$$

For $a$ and $b$ in the set $\{0, \pm 1, \pm 2, \pm 3\}$, let

$$
m+n=7 M+a, \quad m-6 n=7 N+b,
$$

from which it follows that

$$
m=6 M+N+(6 a+b) / 7, \quad n=M-N+(a-b) / 7 .
$$

It follows easily that $a=b$, and so $m=6 M+N+a$ and $n=M-N$, where $-3 \leq a \leq 3$. Thus, there is a one-to-one correspondence between the set of all pairs of integers $(m, n),-\infty<m, n<\infty$, and triples of integers $(M, N, a),-\infty<M, N<\infty,-3 \leq a \leq 3$. By (2.8) and (2.7), we then have

$$
\begin{aligned}
2 \psi & (q) \varphi\left(-q^{3}\right)=f(1, q) f\left(-q^{3},-q^{3}\right) \\
= & \sum_{m, n=-\infty}^{\infty}(-1)^{n} q^{m(m+1) / 2+3 n^{2}} \\
= & \sum_{a=-3}^{3} q^{a(a+1) / 2} \sum_{M=-\infty}^{\infty}(-1)^{M} q^{21 M^{2}+(3+6 a) M} \sum_{N=-\infty}^{\infty}(-1)^{N} q^{N(7 N+1) / 2+a N} \\
= & \sum_{a=-3}^{3} q^{a(a+1) / 2} f\left(-q^{24+6 a},-q^{18-6 a}\right) f\left(-q^{4+a},-q^{3-a}\right) \\
= & 2 f\left(-q^{18},-q^{24}\right) f\left(-q^{3},-q^{4}\right)+2 q f\left(-q^{12},-q^{30}\right) f\left(-q^{2},-q^{5}\right) \\
& +2 q^{3} f\left(-q^{6},-q^{36}\right) f\left(-q,-q^{6}\right)+q^{6} f\left(-q^{42},-1\right) f\left(-q^{7},-1\right) \\
= & f\left(-q^{18},-q^{24}\right) f\left(-q^{3},-q^{4}\right)+2 q f\left(-q^{12},-q^{30}\right) f\left(-q^{2},-q^{5}\right) \\
& +2 q^{3} f\left(-q^{6},-q^{36}\right) f\left(-q,-q^{6}\right) .
\end{aligned}
$$

After dividing both sides by 2 , we obtain the result.

The proofs of the identities (3.3)-(3.7) are very similar to those above. In each case, we find some combinations of pairs $(\alpha, \beta)$ and the corresponding pairs $(\gamma, \delta)$.

5. Proofs of (3.8)-(3.13). Our approach to proving the identities (3.8)-(3.13) uses a generalized formula of H. Schröter [1, pp. 65-72] pro- 
viding a representation for a product of two theta functions as a sum of $m$ products of pairs of theta functions, under certain conditions. An elegant generalization of Schröter's work has been discovered by R. Blecksmith, J. Brillhart, and I. Gerst [4, Theorem 2]. We translate their formula into Ramanujan's notation.

For $\varepsilon \in\{0,1\}$ and $|a b|<1$, define

$$
f_{\varepsilon}(a, b)=\sum_{n=-\infty}^{\infty}(-1)^{\varepsilon n}(a b)^{n^{2} / 2}(a / b)^{n / 2} .
$$

TheOREM 5.1. Let $a, b, c$, and d denote positive numbers with $|a b|,|c d|$ $<1$. Suppose that there exist positive integers $\alpha, \beta$, and $m$ such that

$$
(a b)^{\beta}=(c d)^{\alpha(m-\alpha \beta)} .
$$

Let $\varepsilon_{1}, \varepsilon_{2} \in\{0,1\}$, and define $\delta_{1}, \delta_{2} \in\{0,1\}$ by

$$
\delta_{1} \equiv \varepsilon_{1}-\alpha \varepsilon_{2}(\bmod 2), \quad \delta_{2} \equiv \beta \varepsilon_{1}+p \varepsilon_{2}(\bmod 2),
$$

where $p=m-\alpha \beta$. If $R$ denotes any complete residue system modulo $m$, then

$$
\begin{aligned}
& f_{\varepsilon_{1}}(a, b) f_{\varepsilon_{2}}(c, d) \\
= & \sum_{r \in R}(-1)^{\varepsilon_{2} r} c^{r(r+1) / 2} d^{r(r-1) / 2} f_{\delta_{1}}\left(\frac{a(c d)^{\alpha(\alpha+1-2 r) / 2}}{c^{\alpha}}, \frac{b(c d)^{\alpha(\alpha+1+2 r) / 2}}{d^{\alpha}}\right) \\
& \times f_{\delta_{2}}\left(\frac{(b / a)^{\beta / 2}(c d)^{p(m+1-2 r) / 2}}{c^{p}}, \frac{(a / b)^{\beta / 2}(c d)^{p(m+1+2 r) / 2}}{d^{p}}\right) .
\end{aligned}
$$

Theorem 5.2. The identity (3.8) holds.

Proof. By using (2.11), we see that (3.8) is equivalent to the identity

$$
\begin{aligned}
& f\left(q^{18},-q^{24}\right) f\left(q^{3},-q^{4}\right)+q f\left(-q^{12}, q^{30}\right) f\left(-q^{2}, q^{5}\right) \\
&+q^{3} f\left(q^{6},-q^{36}\right) f\left(q,-q^{6}\right) \\
&=f\left(-q^{2}\right) f\left(-q^{12}\right) \frac{\chi\left(-q^{12}\right)}{\chi(-q)}=\varphi\left(-q^{12}\right) \psi(q) .
\end{aligned}
$$

We apply Theorem 5.1 with $\varepsilon_{1}=1, \varepsilon_{2}=0, a=b=q^{12}, c=q, d=q^{3}$, $\alpha=6, \beta=1$, and $m=7$ to find that

$$
\begin{aligned}
& \varphi\left(-q^{12}\right) \psi(q) \\
&= f\left(-q^{90},-q^{78}\right) f\left(-q^{15},-q^{13}\right)+q f\left(-q^{66},-q^{102}\right) f\left(-q^{11},-q^{17}\right) \\
&+q^{6} f\left(-q^{42},-q^{126}\right) f\left(-q^{7},-q^{21}\right)+q^{15} f\left(-q^{18},-q^{150}\right) f\left(-q^{3},-q^{25}\right) \\
&+q^{28} f\left(-q^{-6},-q^{174}\right) f\left(-q^{-1},-q^{29}\right)+q^{45} f\left(-q^{-30},-q^{198}\right) f\left(-q^{-5},-q^{33}\right) \\
&+q^{66} f\left(-q^{-54},-q^{222}\right) f\left(-q^{-9},-q^{37}\right)
\end{aligned}
$$




$$
\begin{aligned}
= & f\left(-q^{90},-q^{78}\right) f\left(-q^{15},-q^{13}\right)+q f\left(-q^{66},-q^{102}\right) f\left(-q^{11},-q^{17}\right) \\
& +q^{6} f\left(-q^{42},-q^{126}\right) f\left(-q^{7},-q^{21}\right)+q^{15} f\left(-q^{18},-q^{150}\right) f\left(-q^{3},-q^{25}\right) \\
& +q^{21} f\left(-q^{162},-q^{6}\right) f\left(-q,-q^{27}\right)+q^{10} f\left(-q^{138},-q^{30}\right) f\left(-q^{23},-q^{5}\right) \\
& +q^{3} f\left(-q^{19},-q^{9}\right) f\left(-q^{114},-q^{54}\right),
\end{aligned}
$$

where we applied (2.6) six times in the last equality. By (2.13) with $a=q^{3}$, $b=-q^{4}, n=2$, then with $a=-q^{2}, b=q^{5}, n=2$, and finally with $a=q$, $b=-q^{6}, n=2$, we obtain

$$
\begin{gathered}
f\left(q^{3},-q^{4}\right)=f\left(-q^{13},-q^{15}\right)+q^{3} f\left(-q^{27},-q\right), \\
f\left(-q^{2}, q^{5}\right)=f\left(-q^{11},-q^{17}\right)-q^{2} f\left(-q^{25},-q^{3}\right), \\
f\left(q,-q^{6}\right)=f\left(-q^{9},-q^{19}\right)+q f\left(-q^{23},-q^{5}\right) .
\end{gathered}
$$

Replacing $q$ by $q^{6}$ in each of (5.5), (5.6), and (5.7), we obtain

$$
\begin{aligned}
f\left(q^{18},-q^{24}\right) & =f\left(-q^{78},-q^{90}\right)+q^{18} f\left(-q^{162},-q^{6}\right) \\
f\left(q^{12},-q^{30}\right) & =f\left(-q^{66},-q^{102}\right)-q^{12} f\left(-q^{150},-q^{18}\right) \\
f\left(q^{6},-q^{36}\right) & =f\left(-q^{54},-q^{114}\right)+q^{6} f\left(-q^{138},-q^{30}\right) .
\end{aligned}
$$

Return to (5.4) and substitute each of the equalities above to deduce that

$$
\begin{aligned}
& \varphi\left(-q^{12}\right) \psi(q)-\left\{f\left(q^{18},-q^{24}\right) f\left(q^{3},-q^{4}\right)+q f\left(-q^{12}, q^{30}\right) f\left(-q^{2}, q^{5}\right)\right. \\
& \left.+q^{3} f\left(q^{6},-q^{36}\right) f\left(q,-q^{6}\right)\right\} \\
= & -q^{3} f\left(-q^{27},-q\right) f\left(-q^{78},-q^{90}\right)-q^{18} f\left(-q^{13},-q^{15}\right) f\left(-q^{6},-q^{162}\right) \\
& +q^{3} f\left(-q^{25},-q^{3}\right) f\left(-q^{66},-q^{102}\right)+q^{13} f\left(-q^{11},-q^{17}\right) f\left(-q^{18},-q^{150}\right) \\
& -q^{4} f\left(-q^{23},-q^{5}\right) f\left(-q^{54},-q^{114}\right)-q^{9} f\left(-q^{9},-q^{19}\right) f\left(-q^{30},-q^{138}\right) \\
& +q^{6} f\left(-q^{42},-q^{126}\right) f\left(-q^{7},-q^{21}\right) .
\end{aligned}
$$

We now use Theorem 5.1 again, but now with $\varepsilon_{1}=1, \varepsilon_{2}=0, a=1, b=q^{24}$, $c=q, d=q^{3}, \alpha=6, \beta=1$, and $m=7$, to find that

$$
\begin{aligned}
& q^{3} f\left(-1,-q^{24}\right) \psi(q) \\
& =q^{3} f\left(-q^{78},-q^{90}\right) f\left(-q^{27},-q\right)+q^{4} f\left(-q^{54},-q^{114}\right) f\left(-q^{23},-q^{5}\right) \\
& \quad+q^{9} f\left(-q^{30},-q^{138}\right) f\left(-q^{19},-q^{9}\right)+q^{18} f\left(-q^{6},-q^{162}\right) f\left(-q^{15},-q^{13}\right) \\
& \quad+q^{31} f\left(-q^{-18},-q^{186}\right) f\left(-q^{11},-q^{17}\right)+q^{48} f\left(-q^{-42},-q^{210}\right) f\left(-q^{7},-q^{21}\right) \\
& \quad+q^{69} f\left(-q^{-66},-q^{234}\right) f\left(-q^{-3},-q^{25}\right)
\end{aligned}
$$




$$
\begin{aligned}
= & q^{9} f\left(-q^{30},-q^{138}\right) f\left(-q^{9},-q^{19}\right)+q^{18} f\left(-q^{6},-q^{162}\right) f\left(-q^{13},-q^{15}\right) \\
& -q^{13} f\left(-q^{11},-q^{17}\right) f\left(-q^{150},-q^{18}\right)-q^{6} f\left(-q^{42},-q^{126}\right) f\left(-q^{7},-q^{21}\right) \\
& +q^{3} f\left(-q^{78},-q^{90}\right) f\left(-q,-q^{27}\right)+q^{4} f\left(-q^{5},-q^{23}\right) f\left(-q^{54},-q^{114}\right) \\
& -q^{3} f\left(-q^{3},-q^{25}\right) f\left(-q^{66},-q^{102}\right),
\end{aligned}
$$

after several applications of (2.6). The product on the far left side of the above equality equals 0 . Hence we complete the proof.

In a similar way, we can obtain the remaining identities (3.9)-(3.13). If we set $m=7$ in any case, and choose the different parameters $a, b, c, d, \alpha$, $\beta$, then by similar arguments, the proofs are completed.

6. Proofs of (3.14) $-(\mathbf{3 . 2 9})$. The proofs in this section rely strongly on the results of Rogers [10] and Bressoud [5]. We adopt Bressoud's notation, except that we use $q^{n / 24} f\left(-q^{n}\right)$ instead of $P_{n}$, and the variable $q$ instead of $x$. Let $g_{\alpha}^{(p, n)}$ and $\Phi_{\alpha, \beta, m, p}$ be defined as follows:

$$
:=q^{\alpha\left(\frac{12 n^{2}-12 n+3-p}{24 p}\right)} \prod_{r=0}^{\infty} \frac{\left(1-\left(q^{\alpha}\right)^{p r+(p-2 n+1) / 2}\right)\left(1-\left(q^{\alpha}\right)^{p r+(p+2 n-1) / 2}\right)}{\prod_{k=1}^{p-1}\left(1-\left(q^{\alpha}\right)^{p r+k}\right)},
$$

for any positive odd integer $p$, integer $n$, and natural number $\alpha$, and

$$
\Phi_{\alpha, \beta, m, p}:=\sum_{n=1}^{p} \sum_{r, s=-\infty}^{\infty}(-1)^{r+s} q^{\frac{1}{2}\left\{p \alpha\left(r+m \frac{2 n-1}{2 p}\right)^{2}+p \beta\left(s+\frac{2 n-1}{2 p}\right)^{2}\right\}},
$$

where $\alpha, \beta$, and $p$ are natural numbers, and $m$ is an odd positive integer. Then we can immediately obtain the following propositions.

Proposition 6.1 ([5, (2.12) and (2.13)]).

$$
g_{\alpha}^{(5,1)}=q^{-\alpha / 60} G\left(q^{\alpha}\right), \quad g_{\alpha}^{(5,2)}=q^{11 \alpha / 60} H\left(q^{\alpha}\right) .
$$

Proposition 6.2. We have

$$
\begin{aligned}
& g_{\alpha}^{(7,1)}=q^{-\alpha / 42} \frac{f\left(-q^{2 \alpha}\right)}{f\left(-q^{\alpha}\right)} A_{\alpha}, \\
& g_{\alpha}^{(7,2)}=q^{5 \alpha / 42} \frac{f\left(-q^{2 \alpha}\right)}{f\left(-q^{\alpha}\right)} B_{\alpha}, \\
& g_{\alpha}^{(7,3)}=q^{17 \alpha / 42} \frac{f\left(-q^{2 \alpha}\right)}{f\left(-q^{\alpha}\right)} C_{\alpha} .
\end{aligned}
$$

Proof. Take $p=7$ and $n=1$ in (6.1). Then

$$
g_{\alpha}^{(7,1)}=q^{\alpha(-1 / 42)} \prod_{r=0}^{\infty} \frac{\left(1-\left(q^{\alpha}\right)^{7 r+3}\right)\left(1-\left(q^{\alpha}\right)^{7 r+4}\right)}{\prod_{k=1}^{6}\left(1-\left(q^{\alpha}\right)^{7 r+k}\right)}
$$




$$
\begin{aligned}
& =q^{-\alpha / 42} \frac{1}{\left(q^{\alpha} ; q^{7 \alpha}\right)_{\infty}\left(q^{2 \alpha} ; q^{7 \alpha}\right)_{\infty}\left(q^{5 \alpha} ; q^{7 \alpha}\right)_{\infty}\left(q^{6 \alpha} ; q^{7 \alpha}\right)_{\infty}} \\
& =q^{-\alpha / 42} \frac{f\left(-q^{2 \alpha}\right)}{f\left(-q^{\alpha}\right)} A_{\alpha}
\end{aligned}
$$

which gives (6.3). Similarly we can prove (6.4) and (6.5).

LEMMA 6.3 ([5, Proposition 5.1]).

$$
\begin{array}{llrl}
g_{\alpha}^{(p, n)}=g_{\alpha}^{(p,-n+1)}, & g_{\alpha}^{(p, n)}=g_{\alpha}^{(p, n-2 p)}, & g_{\alpha}^{(p, n)}=g_{\alpha}^{(p, 2 p-n+1)}, \\
g_{\alpha}^{(p, n)}=-g_{\alpha}^{(p, n-p)}, & g_{\alpha}^{(p, n)}=-g_{\alpha}^{(p, p-n+1)}, & g_{\alpha}^{(p,(p+1) / 2)}=0 .
\end{array}
$$

Theorem 6.4 ([5, Proposition 5.4]). For odd $p>1$,

$$
\Phi_{\alpha, \beta, m, p}=2 q^{(\alpha+\beta) / 24} f\left(-q^{\alpha}\right) f\left(-q^{\beta}\right)\left\{\sum_{n=1}^{(p-1) / 2} g_{\beta}^{(p, n)} g_{\alpha}^{(p,(2 m n-m+1) / 2)}\right\} .
$$

If we use Lemma 6.3 and Theorem 6.4 for $p=5$ and $p=7$, respectively, we then deduce the following useful lemmas.

LEMMA 6.5.

(6.6) $\Phi_{\alpha, \beta, 1,5}=2 q^{\frac{\alpha+\beta}{40}} f\left(-q^{\alpha}\right) f\left(-q^{\beta}\right)\left\{G\left(q^{\beta}\right) G\left(q^{\alpha}\right)+q^{\frac{\alpha+\beta}{5}} H\left(q^{\beta}\right) H\left(q^{\alpha}\right)\right\}$,

$$
\Phi_{\alpha, \beta, 3,5}=2 q^{\frac{9 \alpha+\beta}{40}} f\left(-q^{\alpha}\right) f\left(-q^{\beta}\right)\left\{G\left(q^{\beta}\right) H\left(q^{\alpha}\right)-q^{\frac{-\alpha+\beta}{5}} H\left(q^{\beta}\right) G\left(q^{\alpha}\right)\right\} .
$$

Proof. Apply Theorem 6.4 with $m=1$ and $p=5$; then we get

$$
\Phi_{\alpha, \beta, 1,5}=2 q^{(\alpha+\beta) / 24} f\left(-q^{\alpha}\right) f\left(-q^{\beta}\right)\left\{g_{\beta}^{(5,1)} g_{\alpha}^{(5,1)}+g_{\beta}^{(5,2)} g_{\alpha}^{(5,2)}\right\} .
$$

By Proposition 6.1, we then have

$$
\begin{aligned}
\Phi_{\alpha, \beta, 1,5} & =2 q^{\frac{\alpha+\beta}{24}} f\left(-q^{\alpha}\right) f\left(-q^{\beta}\right)\left\{q^{\frac{-(\alpha+\beta)}{60}} G\left(q^{\beta}\right) G\left(q^{\alpha}\right)+q^{\frac{11(\alpha+\beta)}{60}} H\left(q^{\beta}\right) H\left(q^{\alpha}\right)\right\} \\
& =2 q^{\frac{\alpha+\beta}{40}} f\left(-q^{\alpha}\right) f\left(-q^{\beta}\right)\left\{G\left(q^{\beta}\right) G\left(q^{\alpha}\right)+q^{\frac{\alpha+\beta}{5}} H\left(q^{\beta}\right) H\left(q^{\alpha}\right)\right\}
\end{aligned}
$$

which reduces to (6.6) after simplification. The equality (6.7) can be proved in a similar way by applying Lemma 6.3 , in addition to Theorem 6.4 with $m=3$ and $p=5$ and Proposition 6.1.

LEMMA 6.6.

$$
\begin{aligned}
\Phi_{\alpha, \beta, 1,7}= & 2 q^{(\alpha+\beta) / 56} f\left(-q^{2 \alpha}\right) f\left(-q^{2 \beta}\right) \\
& \times\left\{A_{\beta} A_{\alpha}+q^{(\alpha+\beta) / 7} B_{\beta} B_{\alpha}+q^{(3 \alpha+3 \beta) / 7} C_{\beta} C_{\alpha}\right\}, \\
\Phi_{\alpha, \beta, 3,7}= & 2 q^{(9 \alpha+\beta) / 56} f\left(-q^{2 \alpha}\right) f\left(-q^{2 \beta}\right) \\
& \times\left\{A_{\beta} B_{\alpha}-q^{(2 \alpha+\beta) / 7} B_{\beta} C_{\alpha}-q^{(-\alpha+3 \beta) / 7} C_{\beta} A_{\alpha}\right\}, \\
\Phi_{\alpha, \beta, 5,7}= & 2 q^{(25 \alpha+\beta) / 56} f\left(-q^{2 \alpha}\right) f\left(-q^{2 \beta}\right) \\
& \times\left\{A_{\beta} C_{\alpha}-q^{(-3 \alpha+\beta) / 7} B_{\beta} A_{\alpha}+q^{(-2 \alpha+3 \beta) / 7} C_{\beta} B_{\alpha}\right\} .
\end{aligned}
$$


Proof. Applying Theorem 6.4 with $m=1$ and $p=7$, we have

$$
\Phi_{\alpha, \beta, 1,7}=2 q^{(\alpha+\beta) / 24} f\left(-q^{\alpha}\right) f\left(-q^{\beta}\right)\left\{g_{\beta}^{(7,1)} g_{\alpha}^{(7,1)}+g_{\beta}^{(7,2)} g_{\alpha}^{(7,2)}+g_{\beta}^{(7,3)} g_{\alpha}^{(7,3)}\right\} .
$$

By (6.3) in Proposition 6.2, we then have

$$
\begin{aligned}
\Phi_{\alpha, \beta, 1,7}= & 2 q^{(\alpha+\beta) / 24} f\left(-q^{2 \alpha}\right) f\left(-q^{2 \beta}\right) \\
& \times\left\{q^{-(\alpha+\beta) / 42} A_{\beta} A_{\alpha}+q^{5(\alpha+\beta) / 42} B_{\beta} B_{\alpha}+q^{17(\alpha+\beta) / 42} C_{\beta} C_{\alpha}\right\},
\end{aligned}
$$

which reduces to (6.8) after simplification. The equalities (6.9) and (6.10) can be proved in a similar way by applying Lemma 6.3 , in addition to Theorem 6.4 with $m=3,5$, respectively, and $p=7$, and Proposition 6.2.

Corollary 6.7 ([5, Corollaries 5.5 and 5.6]). If $\Phi_{\alpha, \beta, m, p}$ is defined by (6.2), then

$$
\begin{aligned}
\Phi_{\alpha, \beta, m, 1} & =0, \\
\Phi_{\alpha, \beta, 1,3} & =2 q^{(\alpha+\beta) / 24} f\left(-q^{\alpha}\right) f\left(-q^{\beta}\right) .
\end{aligned}
$$

Corollary 6.8 ([5, Corollary 5.11]). If $\alpha$ and $\beta$ are even positive integers, then

$$
\Phi_{\alpha, \beta, 1,2}=2 q^{(\alpha+\beta) / 16} \frac{f\left(-q^{2 \alpha}\right) f\left(-q^{2 \beta}\right) f\left(-q^{\alpha / 2}\right) f\left(-q^{\beta / 2}\right)}{f\left(-q^{\alpha}\right) f\left(-q^{\beta}\right)} .
$$

Theorem 6.9 ([5, Corollary 7.3]). Let $\alpha_{i}, \beta_{i}, m_{i}, p_{i}$, where $i=1,2$, be positive integers with $m_{1}, m_{2}$ odd. If $\lambda_{1}:=\left(\alpha_{1} m_{1}^{2}+\beta_{1}\right) / p_{1}$ and $\lambda_{2}:=$ $\left(\alpha_{2} m_{2}^{2}+\beta_{2}\right) / p_{2}$, and

$$
\lambda_{1}=\lambda_{2}, \quad \alpha_{1} \beta_{1}=\alpha_{2} \beta_{2}, \quad \alpha_{1} m_{1} \equiv \pm \alpha_{2} m_{2}\left(\bmod \lambda_{1}\right),
$$

then

$$
\Phi_{\alpha_{1}, \beta_{1}, m_{1}, p_{1}}=\Phi_{\alpha_{2}, \beta_{2}, m_{2}, p_{2}} .
$$

Let $\mathbb{N}$ denote the set of positive integers, and $\mathbb{N}_{0}$ the set of nonnegative integers.

Proposition 6.10. For $u \in \mathbb{N}$,

$$
\Phi_{u, 3 u+4,3,3 u+1}=\Phi_{3 u+4, u, 1, u+1} .
$$

Furthermore, the identity (3.14) holds.

Proof. By Theorem 6.9 with

$$
\begin{array}{llll}
\alpha_{1}=u, & \beta_{1}=3 u+4, & m_{1}=3, & p_{1}=3 u+1, \\
\alpha_{2}=3 u+4, & \beta_{2}=u, & m_{2}=1, & p_{2}=u+1,
\end{array}
$$

and thus $\lambda_{1}=4$, we deduce (6.13). In particular, by taking $u=2$ in (6.13) and then by using (6.9) and (6.12), we have

$$
2 q^{1 / 2} f\left(-q^{4}\right) f\left(-q^{20}\right)\left\{A_{10} B_{2}-q^{2} B_{10} C_{2}-q^{4} C_{10} A_{2}\right\}=2 q^{1 / 2} f\left(-q^{10}\right) f\left(-q^{2}\right) .
$$


After dividing both sides by $2 q^{1 / 2} f\left(-q^{4}\right) f\left(-q^{20}\right)$ and replacing $q^{2}$ by $q$, we deduce that

$$
A_{5} B_{1}-q B_{5} C_{1}-q^{2} C_{5} A_{1}=\frac{f\left(-q^{5}\right) f(-q)}{f\left(-q^{10}\right) f\left(-q^{2}\right)}=\chi(-q) \chi\left(-q^{5}\right),
$$

where the last equality comes from (2.11).

Proposition 6.11. For $u \in \mathbb{N}$,

$$
\Phi_{u, 5 u+12,5,5 u+2}=\Phi_{5 u+12, u, 1, u+2} .
$$

Furthermore, the identity (3.15) holds.

Proof. (6.14) follows from Theorem 6.9 with $\lambda_{1}=\lambda_{2}=6$. Furthermore, by putting $u=1$, we have (3.15) by using (6.10) and (6.12).

Proposition 6.12. For $u \in \mathbb{N}$,

$$
\Phi_{u, 3 u+16,3,3 u+4}=\Phi_{3 u+16, u, 1, u+4} .
$$

Furthermore, the identity (3.16) holds.

Proof. Equality (6.15) follows from Theorem 6.9 with $\lambda_{1}=\lambda_{2}=4$. Furthermore, if we let $u=1$ in (6.15) and use (6.9) and Lemma 6.6, we find that

$$
\begin{aligned}
A_{19} B_{1}-q^{3} B_{19} C_{1}-q^{8} C_{19} A_{1} & \\
= & \left\{G(q) G\left(q^{19}\right)+q^{4} H(q) H\left(q^{19}\right)\right\} \chi(-q) \chi\left(-q^{19}\right) .
\end{aligned}
$$

Now, apply the following identity proved by Biagioli [2]:

$$
=\frac{1}{4 \sqrt{q}} \chi^{2}\left(q^{1 / 2}\right) \chi^{2}\left(q^{19 / 2}\right)-\frac{1}{4 \sqrt{q}} \chi^{2}\left(-q^{1 / 2}\right) \chi^{2}\left(-q^{19 / 2}\right)-\frac{q^{2}}{\chi^{2}(-q) \chi^{2}\left(-q^{19}\right)} .
$$

By combining (6.16) with (6.17), we obtain (3.16).

Proposition 6.13. For $u \in \mathbb{N}$,

$$
\Phi_{2, u(u+3), 1, u+2}=\Phi_{u+3,2 u, 1,3} .
$$

Furthermore, the identity (3.17) holds.

Proof. By Theorem 6.9 with $\lambda_{1}=\lambda_{2}=u+1$, we have (6.18). In particular, by putting $u=5$ and then using (6.8) and (6.12), we obtain the identity (3.17), after replacing $q^{2}$ by $q$ and using (2.11).

Proposition 6.14. For $u \in \mathbb{N}$,

$$
\Phi_{5,3 u+1,2 u+1,4 u+3}=\Phi_{1,15 u+5,1,3} .
$$

Furthermore, the identity (3.18) holds.

Proof. By Theorem 6.9 with $\lambda_{1}=\lambda_{2}=5 u+2$, we have (6.19). In particular, if we let $u=1$ and use (6.9) and (6.12), we deduce the identity (3.18) by using (2.11). 
Proposition 6.15. For $u \in \mathbb{N}$,

$$
\Phi_{1, u^{2}+10 u, 5, u+5}=\Phi_{u+10, u, 1,2} .
$$

Furthermore, the identity (3.19) holds.

Proof. By Theorem 6.9 with $\lambda_{1}=\lambda_{2}=u+5$, (6.20) holds. Furthermore, by putting $u=2$ in (6.20) and using (6.10) and Corollary 6.8, we obtain the identity (3.19), after applying (2.11).

Proposition 6.16. For $u \in \mathbb{N}$,

$$
\Phi_{3 u, 3 u+10,3,3 u+1}=\Phi_{9 u+30, u, 1, u+3} .
$$

Furthermore, the identity (3.20) holds.

Proof. By Theorem 6.9 with $\lambda_{1}=\lambda_{2}=10$, (6.21) holds. Furthermore, if we let $u=2$ in (6.21) and use (6.9) and Lemma 6.6, we can find

$$
\begin{aligned}
A_{8} B_{3}-q^{2} B_{8} C_{3}-q^{3} C_{8} A_{3} & \\
& =\left\{G(q) G\left(q^{24}\right)+q^{5} H(q) H\left(q^{24}\right)\right\} \frac{f(-q) f\left(-q^{24}\right)}{f\left(-q^{6}\right) f\left(-q^{16}\right)} .
\end{aligned}
$$

Recall the following identity proved by Bressoud [5]:

$$
G(q) G\left(q^{24}\right)+q^{5} H(q) H\left(q^{24}\right)=\frac{\chi\left(-q^{3}\right) \chi\left(-q^{12}\right)}{\chi(-q) \chi\left(-q^{4}\right)} .
$$

By combining (6.22) with (6.23), and using

$$
\begin{aligned}
\frac{\chi\left(-q^{3}\right) \chi\left(-q^{12}\right)}{\chi(-q) \chi\left(-q^{4}\right)} \frac{f(-q) f\left(-q^{24}\right)}{f\left(-q^{6}\right) f\left(-q^{16}\right)} & =\frac{f\left(-q^{2}\right) f\left(-q^{3}\right) f\left(-q^{8}\right) f\left(-q^{12}\right)}{f\left(-q^{4}\right) f\left(-q^{6}\right) f\left(-q^{6}\right) f\left(-q^{16}\right)} \\
& =\frac{\chi\left(-q^{2}\right) \chi\left(-q^{8}\right)}{\chi\left(q^{3}\right)}
\end{aligned}
$$

we deduce (3.20).

Proposition 6.17. For $u \in \mathbb{N}$,

$$
\Phi_{2, u^{2}+9 u, 3, u+3}=\Phi_{2 u+18, u, 1,3} .
$$

Furthermore, the identity (3.21) holds.

Proof. Equality (6.24) holds by Theorem 6.9 with $\lambda_{1}=\lambda_{2}=u+6$. Furthermore, by putting $u=4$ in (6.24) and using (6.9) and (6.12), we arrive at (3.21), after replacing $q^{2}$ by $q$ and by $(2.11)$.

Proposition 6.18. For $u \in \mathbb{N}$,

$$
\Phi_{u+3,10 u+16,5,7}=\Phi_{2,5 u^{2}+23 u+24,1, u+2} .
$$

Furthermore, the identity (3.22) holds.

Proof. By Theorem 6.9 with $\lambda_{1}=\lambda_{2}=5 u+13$, (6.25) holds. Furthermore, by putting $u=1$ in (6.25) and using (6.10) and (6.12), we have the result by replacing $q^{2}$ by $q$ and using (2.11). 
Proposition 6.19. For $u \in \mathbb{N}$,

$$
\Phi_{2, u^{2}+3 u, 1, u+1}=\Phi_{2 u+6, u, 1,3} .
$$

Furthermore, the identity (3.23) holds.

Proof. Equality (6.26) holds by Theorem 6.9 with $\lambda_{1}=\lambda_{2}=u+2$. Furthermore, by putting $u=6$ in (6.26) and using (6.8) and (6.12), we get (3.23), after replacing $q^{2}$ by $q$.

Proposition 6.20 ([6, Proposition 5.2]). For $u \in \mathbb{N}_{0}$,

$$
\Phi_{1,8 u+7,2 u+3, u+4}=\Phi_{1,8 u+7,2 u+1, u+2} .
$$

Corollary 6.21. The identity (3.24) holds.

Proof. By taking $u=3$ in (6.27) and applying Theorem 6.4, we find that

$$
\begin{aligned}
2 q^{4 / 3} f(-q) f\left(-q^{31}\right) & \left\{g_{31}^{(7,1)} g_{1}^{(7,5)}+g_{31}^{(7,2)} g_{1}^{(7,14)}+g_{31}^{(7,3)} g_{1}^{(7,23)}\right\} \\
& =2 q^{4 / 3} f(-q) f\left(-q^{31}\right)\left\{g_{31}^{(5,1)} g_{1}^{(5,4)}+g_{31}^{(5,2)} g_{1}^{(5,11)}\right\} .
\end{aligned}
$$

Applying Lemma 6.3, Proposition 6.2, and Proposition 6.1, we find that (6.28) $\quad A_{31} C_{1}-q^{4} B_{31} A_{1}+q^{3} C_{31} B_{1}$

$$
=\left\{G\left(q^{31}\right) H(q)-q^{6} H\left(q^{31}\right) G(q)\right\} \frac{f(-q) f\left(-q^{31}\right)}{f\left(-q^{2}\right) f\left(-q^{62}\right)} .
$$

Now apply the following identity proved by Biagioli [2]:

$$
\begin{aligned}
G\left(q^{31}\right) & H(q)-q^{6} H\left(q^{31}\right) H(q) \\
& =\frac{1}{2 q} \chi(q) \chi\left(q^{31}\right)-\frac{1}{2 q} \chi(-q) \chi\left(-q^{31}\right)-\frac{q^{3}}{\chi\left(-q^{2}\right) \chi\left(-q^{62}\right)} .
\end{aligned}
$$

By combining (6.28) with (6.29), and using

$$
\frac{f(-q) f\left(-q^{31}\right)}{f\left(-q^{2}\right) f\left(-q^{62}\right)}=\chi(-q) \chi\left(-q^{31}\right)
$$

we obtain the identity (3.24).

Proposition 6.22. For $u \in \mathbb{N}$,

$$
\Phi_{1, u^{2}+6 u, 3, u+3}=\Phi_{u+6, u, 1,2} .
$$

Furthermore, the identity (3.25) holds.

Proof. Equality (6.30) holds by Theorem 6.9 with $\lambda_{1}=\lambda_{2}=u+3$. Furthermore, if we let $u=4$, we obtain the identity (3.25), by using (6.9), Corollary 6.8, and (2.11).

Proposition 6.23. For $u \in \mathbb{N}$,

$$
\Phi_{u+1,4 u^{2}, 5, u+5}=\Phi_{u, 4 u(u+1), 1, u} .
$$

Furthermore, the identity (3.26) holds. 
Proof. Equality (6.31) holds by Theorem 6.9 with $\lambda_{1}=\lambda_{2}=4 u+5$. If we set $u=2$, we obtain (3.26) by using (6.10), Corollary 6.8, and (2.11).

Proposition 6.24. For $u \in \mathbb{N}$,

$$
\Phi_{u, 48 u, 1,7}=\Phi_{8 u, 6 u, 1,2} \text {. }
$$

Furthermore, the identity (3.27) holds.

Proof. Equality (6.32) holds, by Theorem 6.9 with $\lambda_{1}=\lambda_{2}=7 u$. If we use (6.8) and Corollary 6.8, then we can find

$$
\begin{aligned}
A_{48 u} A_{u}+q^{7 u} B_{48 u} B_{u}+q^{21 u} C_{48 u} C_{u} & =\frac{f\left(-q^{3 u}\right) f\left(-q^{4 u}\right) f\left(-q^{12 u}\right) f\left(-q^{16 u}\right)}{f\left(-q^{2 u}\right) f\left(-q^{6 u}\right) f\left(-q^{8 u}\right) f\left(-q^{96 u}\right)} \\
& =\frac{\chi\left(-q^{3 u}\right) \chi\left(-q^{12 u}\right) \chi\left(-q^{24 u}\right) \chi\left(-q^{48 u}\right)}{\chi\left(-q^{2 u}\right) \chi\left(-q^{8 u}\right)} .
\end{aligned}
$$

The identity (3.27) follows by replacing $q^{u}$ by $q$.

Proposition 6.25. For $u \in \mathbb{N}_{0}$,

$$
\Phi_{1,16 u^{3}+188 u^{2}+568 u+171,2 u+5, u+7}=0 .
$$

Furthermore, the identity (3.28) holds.

Proof. The identity (6.33) follows from Theorem 6.9 with $\lambda_{1}=\lambda_{2}=$ $416 u^{2}+72 u+28$. Furthermore, if we let $u=0$ in (6.33), we can deduce (3.28) by using (6.10) and (6.11).

Proposition 6.26. For $u \in \mathbb{N}_{0}$,

$$
\Phi_{1,16 u^{3}+172 u^{2}+472 u+195,2 u+1, u+7}=0 .
$$

Furthermore, the identity (3.29) holds.

Proof. The equation (6.34) follows from Theorem 6.9 with $\lambda_{1}=\lambda_{2}=$ $16 u^{2}+68 u+28$. Furthermore, by putting $u=0$ in (6.34), we arrive at (3.29) by using (6.8) and (6.11).

7. Applications to partitions. The identities (3.1) and (3.14) yield theorems in the theory of partitions. In this section, we present partition interpretations for them. For simplicity, we adopt the standard notation

$$
\left(a_{1}, \ldots, a_{n} ; q\right)_{\infty}:=\prod_{j=1}^{n}\left(a_{j} ; q\right)_{\infty}
$$

and define

$$
\left(q^{r \pm} ; q^{s}\right)_{\infty}:=\left(q^{r}, q^{s-r} ; q^{s}\right)_{\infty},
$$

where $r$ and $s$ are positive integers and $r<s$.

First, we need the notion of colored partitions: A positive integer $n$ has $k$ colors if there are $k$ copies of $n$ available and all of them are viewed as distinct objects. Partitions of positive integers into parts with colors 
are called colored partitions. For example, if 1 is allowed to have 2 colors, say $\mathrm{r}$ (red), and g (green), then all colored partitions of 2 are $2,1_{\mathrm{r}}+1_{\mathrm{r}}$, $1_{\mathrm{g}}+1_{\mathrm{g}}, 1_{\mathrm{r}}+1_{\mathrm{g}}$. An important fact is that

$$
\frac{1}{\left(q^{u} ; q^{v}\right)_{\infty}^{k}}
$$

is the generating function for partitions of $n$, where all the parts are congruent to $u(\bmod v)$ and have $k$ colors.

THEOREM 7.1. Let $p_{1}(n)$ denote the number of partitions of $n$ into parts not congruent to $\pm 4, \pm 7, \pm 10(\bmod 21)$ and parts congruent to $\pm 9(\bmod 21)$ with 2 colors. Let $p_{2}(n)$ denote the number of partitions of $n$ into parts not congruent to $\pm 2, \pm 5, \pm 7(\bmod 21)$ and parts congruent to $\pm 6(\bmod 21)$ with 2 colors. Let $p_{3}(n)$ denote the number of partitions of $n$ into parts not congruent to $\pm 1, \pm 7, \pm 8(\bmod 21)$ and parts congruent to $\pm 3(\bmod 21)$ with 2 colors. Then, for any positive integer $n \geq 1$,

$$
p_{1}(n)=p_{2}(n-1)+p_{3}(n) .
$$

Proof. The identity (3.1) is equivalent to

$$
\begin{aligned}
& \text { 1) } \frac{1}{\left(q^{1 \pm}, q^{2 \pm} ; q^{7}\right)_{\infty}\left(q^{3 \pm}, q^{9 \pm} ; q^{21}\right)_{\infty}} \\
& -\frac{q}{\left(q^{1 \pm}, q^{3 \pm} ; q^{7}\right)_{\infty}\left(q^{6 \pm}, q^{9 \pm} ; q^{21}\right)_{\infty}}-\frac{1}{\left(q^{2 \pm}, q^{3 \pm} ; q^{7}\right)_{\infty}\left(q^{3 \pm}, q^{6 \pm} ; q^{21}\right)_{\infty}}=0 .
\end{aligned}
$$

Now, rewrite all the products on the left side of (7.1) subject to the common base $q^{21}$, for example, write $\left(q^{2} ; q^{7}\right)_{\infty}$ as $\left(q^{2} ; q^{21}\right)_{\infty}\left(q^{9} ; q^{21}\right)_{\infty}\left(q^{16} ; q^{21}\right)_{\infty}$. Then (7.1) becomes

$$
\begin{aligned}
\frac{1}{\left(q^{1 \pm}, q^{2 \pm}, q^{3 \pm}, q^{5 \pm}, q^{6 \pm}, q^{8 \pm}, q^{9 \pm}, q^{9 \pm} ; q^{21}\right)_{\infty}} \\
-\frac{q}{\left(q^{1 \pm}, q^{3 \pm}, q^{4 \pm}, q^{6 \pm}, q^{6 \pm}, q^{8 \pm}, q^{9 \pm}, q^{10 \pm} ; q^{21}\right)_{\infty}} \\
-\frac{1}{\left(q^{2 \pm}, q^{3 \pm}, q^{3 \pm}, q^{4 \pm}, q^{5 \pm}, q^{6 \pm}, q^{9 \pm}, q^{10 \pm} ; q^{21}\right)_{\infty}}=0 .
\end{aligned}
$$

Note that the three quotients on the left side of (7.2) represent the generating functions for $p_{1}(n), p_{2}(n)$, and $p_{3}(n)$, respectively. Hence, (7.2) is equivalent to

$$
\sum_{n=0}^{\infty} p_{1}(n) q^{n}-q \sum_{n=0}^{\infty} p_{2}(n) q^{n}-\sum_{n=0}^{\infty} p_{3}(n) q^{n}=0,
$$

where we set $p_{1}(0)=p_{2}(0)=p_{3}(0)=1$. Equating coefficients on both sides yields the desired result. 
ExAMPLE. The following table illustrates the case $n=5$ in Theorem 7.1:

\begin{tabular}{lll}
\hline$p_{1}(5)=6$ & $p_{2}(4)=3$ & $p_{3}(5)=3$ \\
\hline 5 & 4 & 5 \\
$3+2$ & $3+1$ & $2+3_{1}$ \\
$3+1+1$ & $1+1+1+1$ & $2+3_{2}$ \\
$2+2+1$ & & \\
$2+1+1+1$ & & \\
$1+1+1+1+1$ & & \\
\hline
\end{tabular}

THEOREM 7.2. Let $p_{1}(n)$ denote the number of partitions of $n$ into parts not congruent to $\pm 2, \pm 7, \pm 9, \pm 12, \pm 14, \pm 16(\bmod 35)$ and parts congruent to $\pm 10(\bmod 35)$ with 2 colors. Let $p_{2}(n)$ denote the number of partitions of $n$ into parts not congruent to $\pm 1, \pm 6, \pm 7, \pm 8, \pm 13, \pm 14(\bmod 35)$ and parts congruent to $\pm 5(\bmod 35)$ with 2 colors. Let $p_{3}(n)$ denote the number of partitions of $n$ into parts not congruent to $\pm 3, \pm 4, \pm 7, \pm 11, \pm 14, \pm 17$ $(\bmod 35)$ and parts congruent to $\pm 15(\bmod 35)$ with 2 colors. Then, for any positive integer $n \geq 2$,

$$
p_{1}(n)=p_{2}(n-1)+p_{3}(n-2) .
$$

Proof. The identity (3.14) is equivalent to

$$
\begin{aligned}
& \frac{1}{\left(q^{1 \pm}, q^{3 \pm}, q^{4 \pm}, q^{5 \pm}, q^{6 \pm}, q^{8 \pm}, q^{10 \pm}, q^{10 \pm}, q^{11 \pm}, q^{13 \pm}, q^{15 \pm}, q^{17 \pm} ; q^{35}\right)_{\infty}} \\
& -\frac{q}{\left(q^{2 \pm}, q^{3 \pm}, q^{4 \pm}, q^{5 \pm}, q^{5 \pm}, q^{9 \pm}, q^{10 \pm}, q^{11 \pm}, q^{12 \pm}, q^{15 \pm}, q^{16 \pm}, q^{17 \pm} ; q^{35}\right)_{\infty}} \\
& -\frac{q^{2}}{\left(q^{1 \pm}, q^{2 \pm}, q^{5 \pm}, q^{6 \pm}, q^{8 \pm}, q^{9 \pm}, q^{10 \pm}, q^{12 \pm}, q^{13 \pm}, q^{15 \pm}, q^{15 \pm}, q^{16 \pm} ; q^{35}\right)_{\infty}}=1
\end{aligned}
$$

Note that the three quotients on the left side of the above equality represent the generating functions for $p_{1}(n), p_{2}(n)$, and $p_{3}(n)$, respectively. Hence,

$$
\sum_{n=0}^{\infty} p_{1}(n) q^{n}-q \sum_{n=0}^{\infty} p_{2}(n) q^{n}-q^{2} \sum_{n=0}^{\infty} p_{3}(n) q^{n}=1
$$

where we set $p_{1}(0)=p_{2}(0)=p_{3}(0)=1$. Equating coefficients on both sides yields the desired result.

ExAMPLE. The following table illustrates the case $n=7$ in Theorem 7.2:

\begin{tabular}{lll}
\hline$p_{1}(7)=7$ & $p_{2}(6)=3$ & $p_{3}(5)=4$ \\
\hline $6+1$ & $4+2$ & 5 \\
$5+1+1$ & $3+3$ & $2+2+1$ \\
$4+3$ & $2+2+2$ & $2+1+1+1$ \\
$4+1+1+1$ & & $1+1+1+1+1$ \\
$3+3+1$ & \\
$3+1+1+1+1$ & \\
$1+1+1+1+1+1+1$ & & \\
\hline
\end{tabular}


Acknowledgements. I am deeply indebted to Professor Bruce Berndt, my thesis supervisor, for his helpful comments and encouragement. Without his support, I would not have even started this project.

\section{References}

[1] B. C. Berndt, Ramanujan's Notebooks, Part III, Springer, New York, 1991.

[2] A. J. F. Biagioli, A proof of some identities of Ramanujan using modular forms, Glasgow Math. J. 31 (1989), 271-295.

[3] B. J. Birch, A look back at Ramanujan's Notebooks, Math. Proc. Cambridge Philos. Soc. 78 (1975), 73-79.

[4] R. Blecksmith, J. Brillhart, and I. Gerst, Some infinite product identities, Math. Comp. 51 (1988), 301-314.

[5] D. Bressoud, Proof and generalization of certain identities conjectured by Ramanujan, Ph.D. dissertation, Temple University, 1977.

[6] S.-S. Huang, On the Rogers-Ramanujan and Ramanujan-Göllnitz-Gordon continued fractions, Ph.D. dissertation, University of Illinois at Urbana-Champaign, 1997.

[7] S. Ramanujan, Algebraic relations between certain infinite products, Proc. London Math. Soc. 18 (1920), p. xx.

[8] —, Collected Papers, Cambridge Univ. Press, Cambridge, 1927; reprinted by Chelsea, New York, 1962; reprinted by the Amer. Math. Soc., Providence, RI, 2000.

[9] - , The Lost Notebook and Other Unpublished Papers, Narosa, New Delhi, 1988.

[10] L. J. Rogers, On a type of modular relation, Proc. London Math. Soc. (2) 19 (1921), 387-397.

[11] L. J. Slater, Further identities of the Rogers-Ramanujan type, ibid. 54 (1952), 147167.

[12] G. N. Watson, Proof of certain identities in combinatory analysis, J. Indian Math. Soc. 20 (1933), 57-69.

Department of Mathematics

University of Illinois

1409 West Green Street

Urbana, IL 61801, U.S.A.

E-mail: hhahn1@math.uiuc.edu 